\title{
Learning from the big picture
}

S ustainability: valuable goal or illdefined buzzword? Few people would deny that technologies and lifestyles that pollute the environment, deplete irreplaceable resources and threaten to change the climate in unpredictable ways will sooner or later plunge us into crisis. But the problem with seeking 'sustainable' alternatives is that environmental, economic, social and technical considerations are often so deeply entwined that it's not clear where the problem starts or ends.

There's ever more recognition that materials are a central aspect of discussions about sustainability. They could offer greener, friendlier energy technologies, new biomedical solutions, improved telecommunications for remote communities, water purification treatments, and much more. But finding new materials for these tasks is only the start of the process. Markets for 'green' products don't appear overnight, and may be contingent on, for example, supply chains, subsidies, and shifts in public and political attitude.

These are the kinds of complex problems for which we need computational assistance. There is plenty of enthusiasm now for using machine learning to assist and accelerate the process of materials discovery ${ }^{1}$, and increasingly such algorithms are being applied not just to comb 'material space' but to plan and interpret experiments. But the kinds of optimization strategies useful in those contexts are not so different from how computation can convert raw data to useful insights and solutions in quite different areas concerned with sustainability, such as making smart power grids or protecting endangered species. All can benefit from a better understanding of how, for example, to make inferences from observations, to deal with noise, to exploit crowdsourcing and recognize patterns in highdimensional signals.

All this supplies motivation for the ambitious programme called the Computational Sustainability Network (CSN; https://www.compsust.net) ${ }^{2}$, an initiative sponsored by the US National Science Foundation and involving 13 US academic institutions as well as international partners, which aims to nurture this emerging field. The project is led by researchers at Cornell University, headed by computer scientist Carla Gomes, director of Cornell's Institute of Computational Sustainability.

A glance at the topics addressed by the CSN could give an impression of almost unmanageable diversity: they range from landscape conservation in the Andes to predicting weather and food security in Africa and the design of microbial fuel cells. What links them are commonalities in the computational methods used to explore these challenges: machine learning from big data, crowdsourcing and 'citizen science' (for environmental monitoring, say) and the need for optimization strategies in dynamical models. The idea is that, since computational models often involve abstract techniques not tied to any specific domain, tools developed in one sphere can often be valuable in others. That makes the project necessarily highly interdisciplinary.

One major strand is the discovery of new materials for energy generation and storage (fuel cells, batteries, solar and so on) from high-throughput $\mathrm{X}$-ray diffraction experiments ${ }^{3}$.

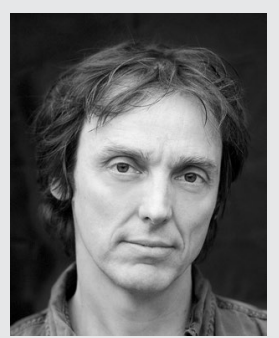

Philip Ball

That goal requires an ability to solve the phase-mapping inverse problem in crystallography, and then to correlate observed structures with functional properties. Both are tasks well suited to machine learning.

These are neat materials challenges in their own right, with no obvious reason to be framed within a broader paradigm of sustainability. But it makes ever more sense to pursue such goals in an integrated manner rather than in isolation. Not only might that lubricate the importation of computational resources and methods developed for other purposes, but - equally importantly - it means that potentially useful innovations may already emerge embedded in the wider picture. The result might then be not just a respectable, citable piece of research, but a technology that will make a difference to the world.

Published online: 23 November 2018

https://doi.org/10.1038/s41563-018-0238-7
References
1. Butler, K. T., Davies, D. W., Cartwright, H., Isayev, O. \& Walsh, A. Nature 559, 547-555 (2018).
2. Gomes, C. et al. Commun. ACM (in the press).
3. Suram, S. K. et al. ACS Combin. Sci. 19, 37-46 (2017). 